TAIWANESE JOURNAL OF MATHEMATICS

Vol. 9, No. 2, pp. 237-243, June 2005

This paper is available online at http://www.math.nthu.edu.tw/tjm/

\title{
ESTIMATES ON SOLUTIONS TO CERTAIN QUASILINEAR EQUATIONS IN DIVERGENCE FORM
}

\author{
Tsang-Hai Kuo
}

\begin{abstract}
The convergence of approximations to solutions of nonlinear elliptic equations is closely related to the structure of the equations. As examples, we examine certain quasilinear elliptic equations with quadratic growth in the gradient defined on bounded domains. $L^{\infty}$ and $H^{1}$ estimates on approximating solutions are performed to deduce the convergence to a solution in $H_{0}^{1}(\Omega) \cap$ $L^{\infty}(\Omega)$. In some cases, $H^{1}$ a priori bound can be derived without referring to $L^{\infty}$ estimate. Furthermore, a $W^{2, p}(\boldsymbol{\Omega})$ bound is also established to deduce the existence of strong solutions in $W^{2, p}(\boldsymbol{\Omega}) \cap W_{0}^{1, p}(\boldsymbol{\Omega})$.
\end{abstract}

\section{INTRODUCTION}

The convergences of approximating solutions to nonlinear elliptic equations in various function spaces are closely related to the structure of equations as well as the constraints on nonlinear terms. As examples, we examine certain quasilinear elliptic problems with quadratic growth in the gradient defined on bounded domains. Let $\Omega$ be a bounded domain in $R^{N}, N \geqslant 3$, which is $C^{1,1}$ diffeomorphic to a ball in $R^{N}$. $L_{v}, L, D_{v}, D$ are the elliptic operators defined by

$$
\begin{aligned}
L_{v} u & =-\sum_{i, j=1}^{N} a_{i j}(x, v) \frac{\partial^{2} u}{\partial x_{i} \partial x_{j}}+c(x, v) u, \\
L u & =L_{u} u, \\
D_{v} u & =-\sum_{i, j=1}^{N} \frac{\partial}{\partial x_{i}} a_{i j}(x, v) \frac{\partial u}{\partial x_{j}}+c(x, v) u, \\
D u & =D_{u} u,
\end{aligned}
$$

Received March 31, 2004; accepted March 8, 2005

Communicated by Chiun-Chuan Chen.

2000 Mathematics Subject Classification: Primary 35D05, 35J25; Secondary 46E35.

Key words and phrases: Quasilinear elliptic problem, Strong solution, $W^{2, p}$ estimate.

Partially supported by the National Science Council of R.O.C. under project NSC 92-2115-M182-001. 
where the coefficients $a_{i j}, c$, and $\frac{\partial a_{i j}}{\partial x_{i}}, \frac{\partial a_{i j}}{\partial r}$ are bounded Carathéodory functions, $c \geqslant \alpha_{0}>0$ for some constant $\alpha_{0}$, and $\sum_{i, j=1}^{N} a_{i j} \xi_{i} \xi_{j} \geqslant \lambda|\xi|^{2}$.

Let $f(x, r, \xi)$ be a Carathéodory function. Consider the following quasilinear elliptic problems

$$
\begin{aligned}
& L u=f(x, u, \nabla u) \text { in } \boldsymbol{\Omega}, \\
& D u=f(x, u, \nabla u) \text { in } \boldsymbol{\Omega},
\end{aligned}
$$

where

$$
|f(x, r, \xi)| \leqslant b(|r|)+h(|r|)|\xi|^{\theta}, 0 \leqslant \theta \leqslant 2,
$$

$b(|r|)$ and $h(|r|)$ are locally bounded functions. When $b(|r|) \equiv C$, it has been shown [2] that Equation (2) has a solution in $H_{0}^{1}(\boldsymbol{\Omega}) \cap L^{\infty}(\boldsymbol{\Omega}) . L^{\infty}, H^{1}$ estimates and then $H^{1}$ convergence to a solution are established in successive steps. In Section 1, we extend to the case $b(|r|)=o(|r|)$. We follow the similar steps to show the existence result of solutions in $H_{0}^{1}(\boldsymbol{\Omega}) \cap L^{\infty}(\boldsymbol{\Omega})$. In case that $|f(x, r, \xi)| \leqslant b(|r|)\left(1+|\xi|^{2}\right)$, $H^{1}$ a priori bound can be derived without refering to the $L^{\infty}$ estimate. If $0 \leqslant \theta<2$, and the oscillations of $a_{i j}(x, r)$ with respect to $r$ are sufficiently small, we shall prove in Section 2 that there exists a solution in $W^{2, p}(\boldsymbol{\Omega}) \cap W_{0}^{1, p}(\boldsymbol{\Omega}), 1 \leq p<\infty$.

\section{2. $H^{1}$ Estimate and the Existence of Solution}

Let $f_{n}$ be the truncation of $f_{n}$ by $\pm n$. Consider the approximating equation

$$
D u=f_{n}(x, u, \nabla u) \text { in } \Omega \text {. }
$$

Notice that the map $v \in H_{0}^{1}(\boldsymbol{\Omega}) \rightarrow f_{n}(x, v, \nabla v)$ is bounded and for every $v \in$ $H_{0}^{1}(\boldsymbol{\Omega})$ there exists a unique $w \in H_{0}^{1}(\boldsymbol{\Omega})$ satisfying $D_{v} w=f_{n}(v, \nabla v)$. The map $v \in H_{0}^{1}(\boldsymbol{\Omega}) \rightarrow w \in H_{0}^{1}(\boldsymbol{\Omega})$ satisfies the hypotheses of Schauder Fixed Point Theorem, so there exists a solution $u_{n} \in H_{0}^{1}(\boldsymbol{\Omega})$ such that

$D u_{n}=f\left(x, u_{n}, \nabla u_{n}\right)$. Moreover, by the weak maximum principle, $\left\|u_{n}\right\|_{L^{\infty}} \leqslant$ $\frac{n}{\alpha_{0}}$.

Now we proceed to the $H^{1}$ estimate of the solutions $\left(u_{n}\right)$. Set $E_{n}=e^{t u_{n}^{2}}$, $v_{n}=E_{n} u_{n}$. Then $\frac{\partial v_{n}}{\partial x_{i}}=E_{n} \frac{\partial u_{n}}{\partial x_{i}}+2 t E_{n} u_{n}^{2} \frac{\partial u_{n}}{\partial x_{i}}$.

Theorem 1. Assume that $f(x, r, \xi) \leqslant b(|r|)\left(1+\left.\xi\right|^{2}\right)$ and $b(|r|)=o(|r|)$. Then the approximating solutions $\left(u_{n}\right)$ are $H^{1}$-bounded.

Proof. Let $\varepsilon$ be given. Since $b(|r|)=o(|r|)$, there exists $C$ and $K>0$ such that $b(|r|) \leqslant C$ for $|r| \leqslant K$ and $b(|r|) \leqslant \varepsilon|r|$ for $|r| \geqslant K$. Denote $\boldsymbol{\Omega}_{n_{1}}=$ $\left\{x|| u_{n}(x) \mid \leqslant K\right\}, \boldsymbol{\Omega}_{n_{2}}=\boldsymbol{\Omega} \backslash \boldsymbol{\Omega}_{n_{1}}$. 
Multiply the test function $v_{n}$ on both sides of Equation (4),

$$
\begin{aligned}
& \sum \int E_{n} a_{i j} \frac{\partial u_{n}}{\partial x_{i}} \frac{\partial u_{n}}{\partial x_{j}}+2 t \sum \int E_{n} u_{n}^{2} a_{i j} \frac{\partial u_{n}}{\partial x_{i}} \frac{\partial u_{n}}{\partial x_{j}}+\int E_{n} c u_{n}^{2} \\
& =\int f_{n}\left(x, u_{n}, \nabla u_{n}\right) E_{n} u_{n} .
\end{aligned}
$$

By the ellipticity, one has

$$
\begin{aligned}
& \lambda \int_{\Omega} E_{n}\left|\nabla u_{n}\right|^{2}+2 t \lambda \int_{\Omega} E_{n} u_{n}^{2}\left|\nabla u_{n}\right|^{2}+\alpha_{0} \int_{\Omega} E_{n} u_{n}^{2} \\
\leqslant & \int_{\Omega}\left|f_{n}\left(x, u_{n}, \nabla u_{n}\right)\right| E_{n}\left|u_{n}\right| \\
\leqslant & \int_{\Omega}\left(b\left(\left|u_{n}\right|\right)\left(1+\left|\nabla u_{n}\right|^{2}\right) E_{n}\left|u_{n}\right|\right. \\
\leqslant & \int_{\boldsymbol{\Omega}_{n_{1}}} C e^{\mathrm{tK}} K+\varepsilon \int_{\boldsymbol{\Omega}_{n_{2}}} E_{n}\left|u_{n}\right|^{2}+\int_{\boldsymbol{\Omega}_{n_{1}}} C\left|\nabla u_{n}\right|^{2} E_{n}\left|u_{n}\right| \\
& +\varepsilon \int_{\boldsymbol{\Omega}_{n_{2}}}\left|\nabla u_{n}\right|^{2} E_{n}\left|u_{n}\right|^{2} .
\end{aligned}
$$

Use Young's inequality, the right-hand side is less than

$$
\begin{aligned}
M & +\varepsilon \int_{\Omega} E_{n}\left|u_{n}\right|^{2}+\frac{\lambda}{2} \int_{\Omega} E_{n}\left|\nabla u_{n}\right|^{2}+\frac{C^{2}}{2 \lambda} \int_{\Omega} E_{n}\left|u_{n}\right|^{2}\left|\nabla u_{n}\right|^{2} \\
& +\varepsilon \int_{\Omega_{n_{2}}}\left|\nabla u_{n}\right|^{2} E_{n}\left|u_{n}\right|^{2} .
\end{aligned}
$$

Choose $\varepsilon=\frac{\alpha_{0}}{2}, t>\frac{1}{4 \lambda^{2}}\left(C^{2}+\alpha_{0} \lambda\right)$ and move the last four terms of (7) to (5), we obtain

$$
\int\left|\nabla u_{n}\right|^{2} \leqslant \int E_{n}\left|\nabla u_{n}\right|^{2} \leqslant \frac{2}{\lambda} M
$$

Finally, by the Poicaré inequality, $\left\|u_{n}\right\|_{H^{1}} \leqslant M^{\prime}$.

Remark 1. The Proof of Theorem 1 indicates that $H^{1}$ bound can be deduced independently without employing $L^{\infty}$ bound.

Consider now Equation (2) with $f$ satisfying (3) when $b(|r|)=C$, an $L^{\infty}$ estimate was performed in [2] by a sort of of weak maximum principle" method. Assume that $b(|r|)=o(|r|)$. Then for every $\varepsilon, \alpha_{0}>\varepsilon>0$, there exists a constant $C>0$ such that

$$
|f(x, r, \xi)| \leqslant C+\varepsilon(|r|)+h(|r|)|\xi|^{2} .
$$


Denote $z_{n}=u_{n}-\frac{C}{\alpha_{0}-\varepsilon}, e_{n}=\exp \left(t\left|z_{n}^{+}\right|^{2}\right)$ and $v_{n}=e_{n} z_{n}^{+}$. Following the demonstration in [2], we multiply the test function $v_{n}$ on both sides of Equation (2.15) in [2, page 28]. Observe that the additional integral $\varepsilon \int\left|z_{n}\right| v_{n}=\varepsilon \int e_{n}\left|z_{n}^{+}\right|^{2}$ on the right-hand side is dominated by $\int C(u)\left|u_{n}\right| v_{n} \geqslant \alpha_{0} \int e_{n}\left|z_{n}^{+}\right|^{2}$ on the left. Thus, from the estimate in [2], $u_{n} \leqslant \frac{C}{\alpha_{0}-\varepsilon}$ for all $n$. In a similar way, one gets $-\frac{C}{\alpha_{0}-\varepsilon} \leqslant u_{n}$ by using $z_{n}=-\frac{C}{\alpha_{0}-\varepsilon}-u_{n}$. Therefore, one concludes that

Lemma 1. Let $f$ be given in (3) with $b(|r|)=o(|r|)$. Then the approximating solutions $\left(u_{n}\right)$ in (4) are $L^{\infty}$-bounded. Moreover, iff satisfies (8) then $\left\|u_{n}\right\|_{L^{\infty}} \leqslant$ $\frac{C}{\alpha_{0}-\varepsilon}$.

Once an $L^{\infty}$ bound is established, $H^{1}$ bound for $\left(u_{n}\right)$ can be further established.

Lemma 2. Let $f$ be given as in (3) with $b(|r|)=o(|r|)$. Then $\left(u_{n}\right)$ is $H^{1}$ bounded.

Proof. Notice that $\left(u_{n}\right)$ is $L^{\infty}$-bounded and hence $h\left(\left|u_{n}(x)\right|\right) \leqslant C_{1}$ a.e. for some constant $C_{1}$.

If one replaces (8) for (6) in the proof of Theorem 1, then

$$
\begin{aligned}
& \int_{\Omega}\left|f\left(x, u_{n}, \nabla u_{n}\right)\right| E_{n}\left|u_{n}\right| \\
\leqslant & \int_{\Omega}\left(b\left|u_{n}\right|+h\left(\left|u_{n}\right|\left|\nabla u_{n}\right|^{2}\right)\right) E_{n}\left|u_{n}\right| \\
\leqslant & \int_{\Omega}\left(C+\varepsilon\left|u_{n}\right|+C_{1}\left|\nabla u_{n}\right|^{2}\right) E_{n}\left|u_{n}\right| \\
\leqslant & M+\varepsilon \int_{\Omega} E_{n}\left|u_{n}\right|^{2}+\frac{\lambda}{2} \int_{\Omega} E_{n}\left|\nabla u_{n}\right|^{2}+\frac{C_{1}^{2}}{2 \lambda} \int_{\Omega} E_{n}\left|u_{n}\right|^{2}\left|\nabla u_{n}\right|^{2} .
\end{aligned}
$$

Choose $\varepsilon=\frac{\alpha_{0}}{2}$ and $t>\frac{C_{1}^{2}}{4 \lambda^{2}}$, it follows from the proof of Theorem 1 that $\left(u_{n}\right)$ is $H^{1}$-bounded.

Together with Theorem 1, one can follow the steps in [2] to show that there exists a subsequence relabeled as $\left(u_{n}\right)$, such that $u_{n} \rightarrow u$ in $H^{1}(\boldsymbol{\Omega})$ and $f_{n}\left(x, u_{n}, \nabla u_{n}\right) \rightarrow$ $f(x, u, \nabla u)$ in $L^{1}(\Omega)$. Therefore, by passing to the limit, one concludes that $u \in$ $H_{0}^{1}(\Omega) \cap L^{\infty}(\Omega)$ is a solution to $(2)$.

Theorem 2. Let $f$ be given as in (3) with $b(|r|)=o(|r|)$. Then there exists a solution $u \in H_{0}^{1}(\Omega) \cap L^{\infty}(\Omega)$ to $(2)$. 


\section{Strong Solutions to Equations in Divergence Form}

In this section, we examine the existence of $W^{2, p}(\Omega) \cap W_{0}^{1, p}(\Omega)$ solutions to the quasilinear equations in divergence form,

$$
\begin{aligned}
D \mathrm{u} & =-\sum_{i, j=1}^{N} \frac{\partial}{\partial x_{i}} a_{i j}(x, u) \frac{\partial u}{\partial x_{j}}+C(x, u) u \\
& =f(x, u, \nabla u) \text { in the sense of distribution } \mathfrak{O}(\Omega),
\end{aligned}
$$

where the cofficients $a_{i j} \in C^{0,1}(\bar{\Omega} \times R)$, and

$$
|f(x, r, \xi)| \leqslant C_{0}+h(|r|)|\xi|^{\theta}, 0 \leq \theta<2
$$

For a fixed point $x \in R^{N}$, we denote osc $a_{i j}(x, r)$ the oscillation of $a_{i j}(x, r)$ with respect to $r$, that is, osc $a_{i j}(x, r) \equiv \sup \left\{\left|a_{i j}\left(x, r_{1}\right)-a_{i j}\left(x, r_{2}\right)\right|: r_{1}, r_{2} \in R\right\}$, and osc $a_{i j}(x, r)=\max _{1 \leq i, j \leq N}$ ose $a_{i j}(x, r)$.

For operators $L_{v}$, we quote the following result from [5, p.191].

Lemma 3. Let $\Omega$ be a bounded domain in $R^{N}$ which is $C^{1,1}$ diffeomorphic to a ball in $R^{N}$, and the cofficients $a_{i j} \in C^{0,1}(\bar{\Omega} \times R),\left|a_{i j}\right|,|c| \leqslant \Lambda$, where $\Lambda$ is a positive constant, $i, j=1, \ldots, N$. Assume that osc $a_{i j}(x, r)$ is sufficiently small with respect to $r$ and uniformly for $x \in \Omega$. Then if $u \in W^{2, p}(\Omega) \cap W_{0}^{1, p}(\Omega)$ and $L_{v} u \in L^{p}(\Omega), 1<p<\infty$. One has the estimate

$$
\|u\|_{W^{2, p}(\Omega)} \leqslant C\left(\left\|L_{v} u\right\|_{L^{p}(\Omega)}+\|u\|_{L^{p}(\Omega)}\right),
$$

where $C$ is a constant (independent of $v$ ) dependent on $N, p, \lambda, \Lambda, \partial \Omega$, and $\Omega$, the diffeomorphism and the mpduli of continuity of $a_{i j}(x, r)$ with respect to $x$ in $\bar{\Omega}$.

Consider now Equation (1). Suppose $f(x, r, \xi)$ is bounded. For $v \in W^{2, p}(\Omega) \cap$ $W_{0}^{1, p}(\Omega)$, the Dirichlet problem $L_{v} u=f(x, v, \nabla u)$ has a unique solution $u \in$ $W^{2, p}(\Omega) \cap W_{0}^{1, p}(\Omega)$ and by Theorem 2 ,

$$
\|u\|_{W^{2, p}(\Omega)} \leqslant C\left(\|u\|_{L^{p}(\Omega)}+\|f(x, v, \nabla v)\|_{L^{p}(\Omega)}\right)
$$

An application of the Weak Maximum Principle of A.D Aleksendrov [4, p.220] together with the Schauder Fixed Point Theorem implies that

Lemma 4. Suppose that $f(x, r, \xi)$ is bounded. Then for each $1 \leq p<\infty$, there exists a solution $u \in W^{2, p}(\Omega) \cap W_{0}^{1, p}(\Omega)$.

Proof. See[5. p118]. 
Let

$$
\begin{gathered}
\tilde{f}(x, r, \xi)=f(x, r, \xi)+\sum \frac{\partial a_{i j}}{\partial x_{i}} \xi_{i}+\sum \frac{\partial a_{i j}}{\partial r} \xi_{i} \xi_{j}, \\
\widetilde{g}_{n}(x, r, \xi)=\tilde{f}_{n}(x, r, \xi)+\sum \frac{\partial a_{i j}}{\partial x_{i}} \xi_{i}+\sum \frac{\partial a_{i j}}{\partial r} \xi_{i} \xi_{j},
\end{gathered}
$$

In view of Lemma 4 , there exists a $W^{2, p}(\Omega) \cap W_{0}^{1, p}(\Omega)$ solution $u_{n}$, satisfying

$$
L u_{n}=\widetilde{f}_{n}\left(x, u_{n}, \nabla u_{n}\right)
$$

or equivalently,

$$
D u_{n}=\tilde{g}_{n}\left(x, u_{n}, \nabla u_{n}\right) .
$$

Observe that for $\varepsilon>0,\left.\left|\tilde{g}_{n}(x, r, \xi) \leqslant C_{0}+\varepsilon+h_{1}(|r|)\right| \xi\right|^{2}$, where $h_{1}(|r|)$ is a locally bounded function. By Lemma $1,\left\|u_{n}\right\|_{L^{\infty}} \leqslant \frac{C_{0}}{\alpha_{0}}$. One can now proceed to the $W^{2, p}$ estimate and then show the existence of $W^{2, p}(\Omega) \cap W_{0}^{1, p}(\Omega)$ solutions to (9).

Lemma 5. If in addition to the assumption of Lemma 3 and (10), the cofficients $a_{i j}$ are independent of $r$ for $|r| \leqslant \frac{C_{0}}{\alpha_{0}}$. Then there exists a $W^{2, p}$-bounded subsequence of the approximating solutions $\left(u_{n}\right)$ in $W^{2, p}(\Omega) \cap W_{0}^{1, p}(\Omega)$ to Equation (9).

Proof. As described in the above paragraphs, the approximating solutions $\left(u_{n}\right)$ to (13) are $L^{\infty}$-bounded by $\frac{C_{0}}{\alpha_{0}}$. By the assumption that $a_{i j}(x, r)=a_{i j}(x)$ and consequently $\frac{\partial a_{i j}}{\partial r}=0$ for $|r| \leqslant \frac{C_{0}}{\alpha_{0}}$.

$$
\hat{f}\left(x, u_{n}, \nabla u_{n}\right)=f\left(x, u_{n}, \nabla u_{n}\right)-\sum \frac{a_{i j}}{\partial x_{i}}\left(x, u_{n}, \nabla u_{n}\right) \frac{\partial u_{n}}{\partial x_{j}} .
$$

For $\varepsilon>0$, there exists $C_{\varepsilon}>0$ such that $|\xi|^{\theta} \leqslant C_{\varepsilon}+\varepsilon|\xi|^{2}$. Combine with (10),

$$
\left|\hat{f}_{n}\left(x, u_{n}, \nabla u_{n}\right)\right| \leqslant C_{1}+C_{2} \varepsilon\left|\nabla u_{n}\right|^{2},
$$

for some constants $C_{1}, C_{2}$.

In view of the estimate (11), one has

$$
\begin{aligned}
\|u\|_{W^{2, p}} & \leqslant C\left(\left\|u_{n}\right\|_{L^{p}}+\left\|\tilde{f}_{n}\left(x, u_{n}, \nabla u_{n}\right)\right\|_{L^{p}}\right) \\
& \leqslant C_{2}+\varepsilon C_{2}\left\|\nabla u_{n}\right\|_{L^{2 p}}^{2} .
\end{aligned}
$$

Now since $u_{n} \in L^{\infty}(\Omega) \cap W^{2, p}(\Omega)$, from the Interpolation Theorem of GagliardoNirenberg [1, p.194], we obtain

$$
\begin{aligned}
\|u\|_{W^{2, p}} & \leqslant C_{2}+\varepsilon C_{2}^{\prime}\left\|u_{n}\right\|_{W^{2, p}}\left\|u_{n}\right\|_{L^{\infty}} \\
& \leqslant C_{2}+\varepsilon C_{2}^{\prime} \frac{C_{0}}{\alpha_{0}}\left\|u_{n}\right\|_{W^{2, p}} .
\end{aligned}
$$


Hence, by choosing $\varepsilon C_{2}^{\prime} \frac{C_{0}}{\alpha_{0}}=\frac{1}{2}$, we conclude that $\left\|u_{n}\right\|_{W^{2, p}} \leqslant C_{3}$, i.e. $\left(u_{n}\right)$ is $W^{2, p}$-bounded.

The existence of strong solutions can now be concluded from above lemmas. We summerize in our main theorem.

Theorem 3. Let $\Omega$ be a bounded $c^{1.1}$-smooth domain in $R^{N}, N \geqslant 3$. The coefficients $a_{i j}, \frac{\partial a_{i j}}{\partial x_{i}}, \frac{\partial a_{i j}}{\partial x_{i}}$, c are bounded Caratheory functions. Assume that osc $a(x, r)$ is sufficiently small with respect to $r$ and uniformly for $x \in \Omega, a_{i j}$ are independent of $r$ for $|r| \leqslant \frac{C_{0}}{\alpha_{0}}$, and $f$ satisfies (8). Then for each $p, 1 \leqslant p<\infty$, there exists a solution $u \in W^{2, p}(\Omega) \cap W_{0}^{1, p}(\Omega)$ to $(8)$ with $\|u\|_{L^{\infty}} \leqslant \frac{C_{0}}{\alpha_{0}}$.

Proof. By Lemma 5 we get the approximating solutions $\left(u_{n}\right)$ which is $W^{2, p}(\Omega)$ bounded. It follows from the compact imbedding $W^{2, p}(\Omega) \rightarrow W^{1, p}(\Omega)$ that there exists a convergent subsequence in $W^{1, p}(\Omega)$, which is still denoted by $\left(u_{n}\right)$, such that $u_{n} \rightarrow u$ a.e., $\nabla u_{n} \rightarrow \nabla u$ a.e. and $u_{n} \rightarrow u$ in $W^{1, p}(\Omega)$.

Passing to the limit and using Vitali Convergence Theorem, one can show that $L u_{n} \rightarrow L u$ in $\mathfrak{O}(\Omega)$ and $f_{n}\left(x, u_{n}, \nabla u_{n}\right) \rightarrow f(x, u, \nabla u)$ in $L^{1}(\Omega)$. Moreover, since $\left\|u_{n}\right\|_{W^{2, p}} \leqslant M$ and the set

$$
\left\{v \in W^{2, p}(\Omega) \cap W_{0}^{1, p}(\Omega) \mid\left\|u_{n}\right\|_{W^{2, p}} \leqslant M\right\}
$$

is closed in $W_{0}^{1, p}(\Omega)$, the limit $\mathrm{u}$ of $\left(u_{n}\right)$ belong to $W^{2, p}(\Omega) \cap W_{0}^{1, p}(\Omega)$. The existence of solutions in $W^{2, p}(\Omega) \cap W_{0}^{1, p}(\Omega)$ to Problem (9) is now asserted.

\section{REFERENCES}

1. H. Brezis, Analyse Fonctionnelle Théorie et Applications, Masson, Paris, 1983.

2. L. Boccardo, F. Murat and J. P. Puel, Existence de solutions faibles pour des équations elliptiques quasi-linéaires a croissance quadratique, in: Nonlinear Partial Differential Equations and Their Applications, J. L. Lions and H. Brezis eds., Collége de France Seminar, Vol. IV, Research Notes in Math. 84, Pitman, London, 1983, 19-73.

3. L. Boccardo, F. Murat and J. P. Puel, Résultats d'existence pour certains problems elliptiques quasi-linéaires, Ann. Scuola Norm. Sup. Pisa Cl. Sci. 11 (1984).

4. D. Gilbarg and N. S. Trudinger, Elliptic Partial Differential Equations of Second Order, second edition, Springer-Verlag, New York, 1983.

5. T. H. Kuo and Y. J. Chen, Existence of strong solutions to some quasilinear elliptic problems on bounded smooth domains, Taiwanese J. Math. 6 (2002), 187-204.

Tsang-Hai Kuo

Center for General Education,

Chang-Gung University,

Taoyuan 333, Taiwan.

E-mail: thkuo@mail.cgu.edu.tw 